\title{
LOS ITINERARIOS DE APRENDIZAJE MEDIANTE MAPAS CONCEPTUALES COMO RECURSO PARA LA REPRESENTACIÓN DEL CONOCIMIENTO
}

\author{
LEARNING PATH BY USING CONCEPT MAPS AS A RESOURCE FOR \\ KNOWLEDGE REPRESENTATION \\ Bárbara de Benito Crossetti ; barbara.debenito@uib.es \\ Antònia Darder Mesquida ; antonia.darder@uib.es \\ Jesús Salinas Ibáñez; jesus.salinas@uib.es \\ Universitat de les Illes Balears
}

\section{RESUMEN}

Nuestra idea es que los mapas conceptuales pueden utilizarse como organizadores de la secuencia del aprendizaje en forma de lo que denominamos itinerarios de aprendizaje (Salinas, de Benito y Darder, 2011). En este estudio, se diseñó y validó por un grupo de expertos, un itinerario de aprendizaje basado en mapas conceptuales para el estudio de un módulo de contenidos de la asignatura Tecnología Educativa II, para presentarlo posteriormente a dos grupos de alumnos con el fin de obtener resultados sobre su implementación, metodología, la organización acorde con los postulados del aprendizaje significativo, los cambios que puede provocar dicha organización, la flexibilidad que ofrecen al proceso de aprendizaje y el logro de las competencias propuestas.

PALABRAS CLAVE: Itinerarios de aprendizaje, mapas conceptuales, diseño de procesos de aprendizaje, organizador de aprendizaje.

\section{ABSTRACT}

Our idea is that concept maps can be used as organizers of the sequence of learning in the form of what we call learning paths. In this study, a learning path based on concept maps was designed and validated by an expert group, to study a module of the course content Educational Technology II. This route was presented to two groups of students to get results on: 1) Their implementation and the methodology. 2) The organization consistent with the principles of meaningful learning. 3) Changes that the organization can prompt. 4) The available to flexibility the learning process. 5) The achievement of the competencies proposed.

KEYWORDS: Learning paths, concept mapping, learning design, learning organizer. 


\section{INTRODUCCIÓN}

El proyecto de innovación educativa en el cual se enmarca este trabajo se realizó durante los cursos 2009-10 y 2010-11, y se basa en que las instituciones responderán al desafío de la introducción de las TIC en los procesos de enseñanza-aprendizaje, si promueven experiencias innovadoras en dichos procesos, apoyándose en las TIC y "haciendo énfasis en la docencia, en los cambios de estrategias didácticas de los profesores y en los sistemas de comunicación y distribución de los materiales de aprendizaje, es decir, en los procesos de innovación docente" (Salinas, 2004). De esta forma, el rol del docente también cambia, el docente pasa a ser un guía en el aprendizaje del alumno, facilitando el uso de los recursos y herramientas que necesitan para la adquisición de nuevos conocimientos, lo cual también implica el cambio en el rol del alumno, que pasa a formar parte activa en los procesos de enseñanza-aprendizaje. No podemos obviar los cambios metodológicos, teniendo en cuenta que a la hora de tomar decisiones sobre el diseño de la enseñanza, tendremos que tener en cuenta que éstas vienen ligadas a aspectos relacionados con el tipo de institución, el diseño de la enseñanza en sí, y con el alumno y el aprendizaje.

En este nuevo contexto, los alumnos deben aprender a ser críticos con la gran cantidad de información que reciben, y por tanto, como recurso clave deben aprender a organizar la información, ser críticos ante ella y ser capaces de asimilarla y reconceptualizarla de forma creativa (González, 2008). Y es en este proceso de aprendizaje dónde la función del docente es fundamental. En este sentido, cómo estructurar y secuenciar los contenidos de una disciplina de acuerdo a un buen diseño instruccional es una de las cuestiones más importantes en los procesos de enseñanza-aprendizaje en entornos virtuales (de Benito, Salinas, Darder \& Marín, 2011). De esta forma, Reigeluth (1995) plantea la teoría instruccional como la que ha de proveer de una guía para fortalecer la iniciativa y responsabilidad de los alumnos para la construcción de su aprendizaje.

Así, para que el aprendizaje sea significativo, eficiente y eficaz, requiere según Ausubel de: "una estructura cognitiva apropiada del alumno, materiales de aprendizaje significativos, conceptualmente transparentes y una disposición favorable por parte del alumno hacia este tipo de aprendizaje". De esta forma, y para lograr los objetivos educativos específicos debemos conocer las posibilidades de las herramientas de las que disponemos (de Benito, 2000). Es por ello que presentamos al estudiante la información en forma de mapas conceptuales, ya que estos son una potente herramienta para organizar, representar y almacenar el conocimiento. Se basan en un esquema de conceptos y relaciones entre ellos, unidas por proposiciones o palabras y organizadas jerárquicamente, y constituyen una de las principales aplicaciones prácticas de la teoría de Novak sobre el aprendizaje significativo frente al aprendizaje memorístico (Novak, 1998). Los contenidos se organizan en conceptos, y estos tienen asociados recursos que dan una información ampliada sobre ese concepto (que serán vídeos, textos, otros mapas, etc.) y así, el control sobre la navegación lo tiene totalmente el alumno, pues en este tipo de materiales no se puede determinar la manera en que el alumno navega. 
En de Benito, Darder, Salinas \& Cañas (2010) y Salinas, de Benito y Darder (2011), un itinerario de aprendizaje viene a ser un mapa conceptual que nos guía en el aprendizaje sobre un tema presentando una serie de competencias que deben comprenderse, dominarse y demostrarse para entenderlo, y que a diferencia del mapa conceptual convencional que explica el tema (los conceptos y sus relaciones, el qué de un tema), en este caso se ocupa del cómo aprenderlo. Supone, por tanto, una forma de organizar la secuencia de aprendizaje y responde a la necesidad de guía de los alumnos por los contenidos, procesos y actividades, proporcionando, al mismo tiempo, suficiente flexibilidad para que ejerza cierta autonomía en el proceso de aprendizaje.

En este estudio los itinerarios de aprendizaje mediante mapas conceptuales se han utilizado con una doble finalidad:

- Presentación de un itinerario de aprendizaje experto como herramienta para representar, secuenciar y organizar el aprendizaje y sus recursos.

- Creación por parte de los alumnos de su propio itinerario de aprendizaje.

Concretándose los objetivos de investigación en:

1. Diseñar, validar e implementar el itinerario de aprendizaje mediante mapas conceptuales como herramienta para representar, secuenciar y organizar el aprendizaje y sus recursos.

2. Valorar la utilización de los itinerarios de aprendizaje, elaborados por los propios alumnos, como instrumento de apoyo al aprendizaje y para validar y evaluar el proceso de aprendizaje

\section{METOdología}

La investigación se realizó en dos grandes fases, las cuales están divididas en diferentes etapas, siguiendo el proceso de la investigación de desarrollo de Reeves (2000) (Fig. 1).

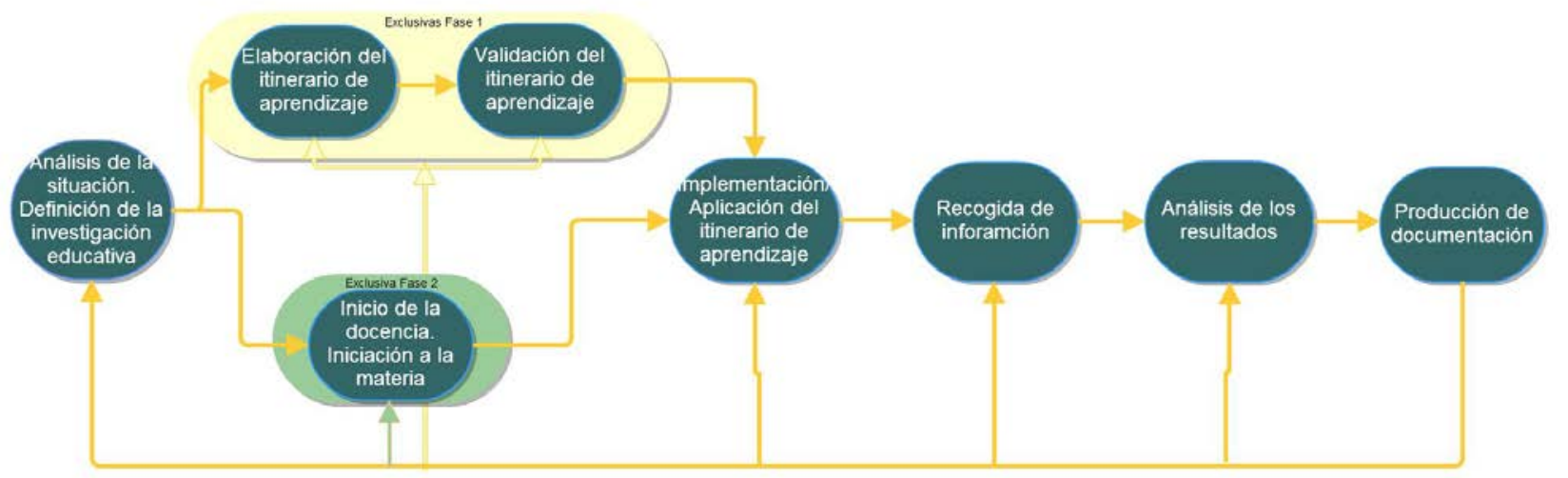

Figura 1. Fases del proceso de investigación de desarrollo (Adaptado de Reeves, 2000) 


\section{Primera fase}

La primera fase consistió en la elaboración, validación e implementación del itinerario de aprendizaje experto.

De acuerdo con el primer objetivo de investigación se prentedía dar respuesta a las cuestiones siguientes: ¿El itinerario de aprendizaje ayuda a los profesores a organizar la asignatura más acorde con los postulados del aprendizaje significativo? ¿El diseño de asignaturas usando Itinerarios de aprendizaje obliga a cambios en la organización de los contenidos, en los objetos de aprendizaje ofrecidos, en las actividades, en la forma de trabajo...? ¿El diseño de asignaturas usando itinerarios de aprendizaje proporciona mayor flexibilidad al proceso de aprendizaje? ¿EI uso de itinerarios de aprendizaje contribuye al logro de las competencias propuestas en mayor grado?

Se implementó con la cohorte de alumnos que cursaban la asignatura de Tecnología Educativa II correspondiente al tercer año de los estudios de Pedagogía (curso 2009-2010). Se eligió el tema de diseño y producción de materiales didácticos multimedia que constituye un módulo fundamental dentro del temario de la asignatura. El trabajo se dividió en 7 etapas:

- Primera. Análisis de la situación. Definición de la investigación educativa.

- Segunda. Elaboración del itinerario de aprendizaje para el Módulo III: "Diseño de materiales multimedia interactivos".

- Tercera. Validación del Itinerario de Aprendizaje por expertos. Una vez elaborado el itinerario se procedió a la validación del mismo por 7 expertos (en elaboración y utilización de mapas conceptuales, en diseño de materiales didácticos multimedia, en docencia en disciplinas similares). Para ello se dió acceso al itinerario a dichos expertos y se les pasó un cuestionario mixto, en el que además de valorar distintos aspectos del mismo, se incluyeron cuestiones abiertas donde se solicitaba señalar los puntos fuertes y débiles además de incluir propuestas para su revisión y mejora. De los 7 expertos se recibió el cuestionario de 5 . Se estudiaron las propuestas de revisión y mejora, incorporando la mayoría de estas, con lo que se obtuvo una versión corregida del 'ITINERARIO PARA LA CREACIÓN DE MATERIALES MULTIMEDIA INTERACTIVOS' (Fig. 2). 


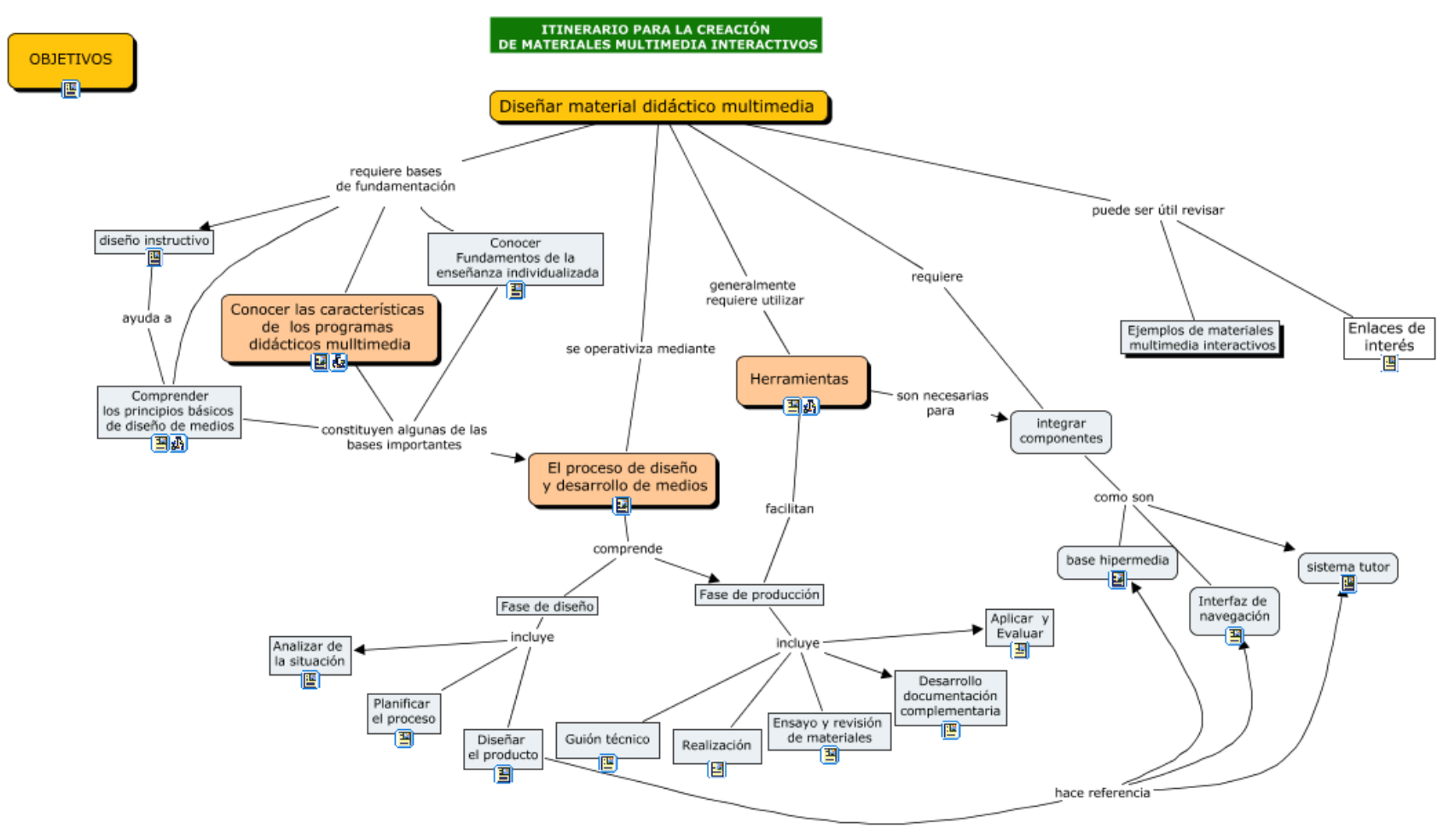

Figura 2. Itinerario para la creación de materiales multimedia interactivos.

- Cuarta. Implementación/Aplicación del itinerario de aprendizaje a los alumnos. Ésta se llevó a cabo de forma secuencial:

o Se realizó una sesión presencial para una breve explicación del itinerario y de las tareas a desarrollar con el mismo.

o Se estableció un plazo de 8 semanas para trabajar de forma autónoma con el itinerario e ir creando un proyecto para la realización de un material multimedia interactivo, por tanto, obliga a la revisión del material.

o Antes de la entrega de dicho proyecto se realiza una sesión teórica presencial para resolver cualquier duda que les pueda haber surgido durante la revisión del itinerario y el proyecto que están realizando.

o Transcurrido el período de trabajo con el itinerario se realizó una prueba escrita de conocimientos teóricos adquiridos en relación con el tema.

o Se les propuso como actividad voluntaria la construcción de su propio itinerario de aprendizaje.

o Durante todo el cuatrimestre, los alumnos debían asistir a clases prácticas presenciales en el Laboratorio de Informática, dirigidas a la adquisición de las destrezas y conocimientos técnicos necesarios para elaborar el material multimedia (proyecto del itinerario de aprendizaje)

o $Y$ finalmente, se presentó en sesión presencial colectiva el material multimedia elaborado.

- Quinta. Recogida de información y evaluación por parte de los usuarios. Los instrumentos utilizados para el análisis de la experiencia se recogen en la tabla 1. 


\begin{tabular}{|l|l|ll|}
\hline Para analizar la perspectiva del profesor & $\begin{array}{l}\text { Para el análisis de las reacciones, los aprendizajes } \\
\text { y la transferencia }\end{array}$ \\
\hline Entrevista al profesor de la la & $\begin{array}{l}\text { Cuestionarios a los alumnos } \\
\text { asignatura, sobre su opinión, los } \\
\text { cambios en la organización de la }\end{array}$ & $\begin{array}{l}\text { Material multimedia elaborado por los } \\
\text { alumnos }\end{array}$ \\
$\begin{array}{l}\text { asignatura, el proceso de de } \\
\text { elaboración del mapa, desarrollo de } \\
\text { estrategias didácticas, ... }\end{array}$ & $\begin{array}{l}\text { Creación de mapas o itinerarios de los alumnos } \\
\text { para estudiar el tema }\end{array}$ \\
& $\begin{array}{l}\text { Prueba escrita } \\
\text { - Entrevistas a los alumnos. (Selección de una } \\
\text { muestra) }\end{array}$ \\
\hline
\end{tabular}

Tabla 1. Instrumentos aplicados en el estudio

En esta primera fase, de los 76 alumnos matriculados en la asignatura, 15 fueron seleccionados para hacerles una entrevista personal, 32 participaron en los cuestionarios y se hizo una entrevista a las profesoras.

- Sexta. Análisis de los resultados.

- Séptima. Producción de documentación.

\section{Segunda Fase}

En la segunda fase se consolidó el itinerario de aprendizaje experto, introduciendo mejoras relacionadas con los resultados obtenidos en la fase anterior y se incorporó la utilización de los itinerarios de aprendizaje, elaborados por los propios alumnos, como instrumento de apoyo al aprendizaje y para validar y evaluar el proceso de aprendizaje.

El estudio se realizó, de igual forma, en la asignatura de Tecnología Educativa II perteneciente al 3er curso de los estudios de pedagogía (curso 2010-11). Al igual que en la primera fase, el itinerario de aprendizaje experto se aplica en el módulo III y su objetivo fue que los alumnos (en grupos de máx. 3 personas) diseñaran y produjeran un material multimedia interactivo. Para ello se trabajaron fundamentos teóricos, etapas de diseño y producción y clases prácticas (de carácter instrumental para el aprendizaje del manejo de diferentes programas para desarrollarlo).

Siguiendo la misma estructura que en la primera parte y el esquema de la FIG. 1, para esta segunda se divide en seis etapas.

- Primera. Análisis de la situación. Definición de la investigación educativa. Modificación del itinerario de aprendizaje y redefinición de la estrategia metodológica en función de la valoración realizada por los participantes de la fase anterior.

- Segunda. Inicio de la docencia. Iniciación a la materia. De acuerdo con los resultados obtenidos en la primera fase se decide introducir un taller sobre la elaboración de mapas conceptuales. Es también en esta etapa cuando se explica a los alumnos el 
trabajo que deben realizar durante todo el cuatrimestre, y por tanto, el itinerario personal de aprendizaje.

- Tercera. Implementación/Aplicación del itinerario de aprendizaje experto al colectivo de alumnos. Siguiendo las recomendaciones obtenidas en la fase 1, se organizó un plan de trabajo con actividades más pautadas y fechas de entrega así como la organización de seminarios presenciales para el seguimiento de las actividades y la solución de problemas en la adquisición de conocimientos teóricos.

- Cuarta. Recogida de información. La recogida de información y datos y para la evaluación de la materia y la investigación se llevaron a cabo a través de los siguientes instrumentos (Tabla 2).

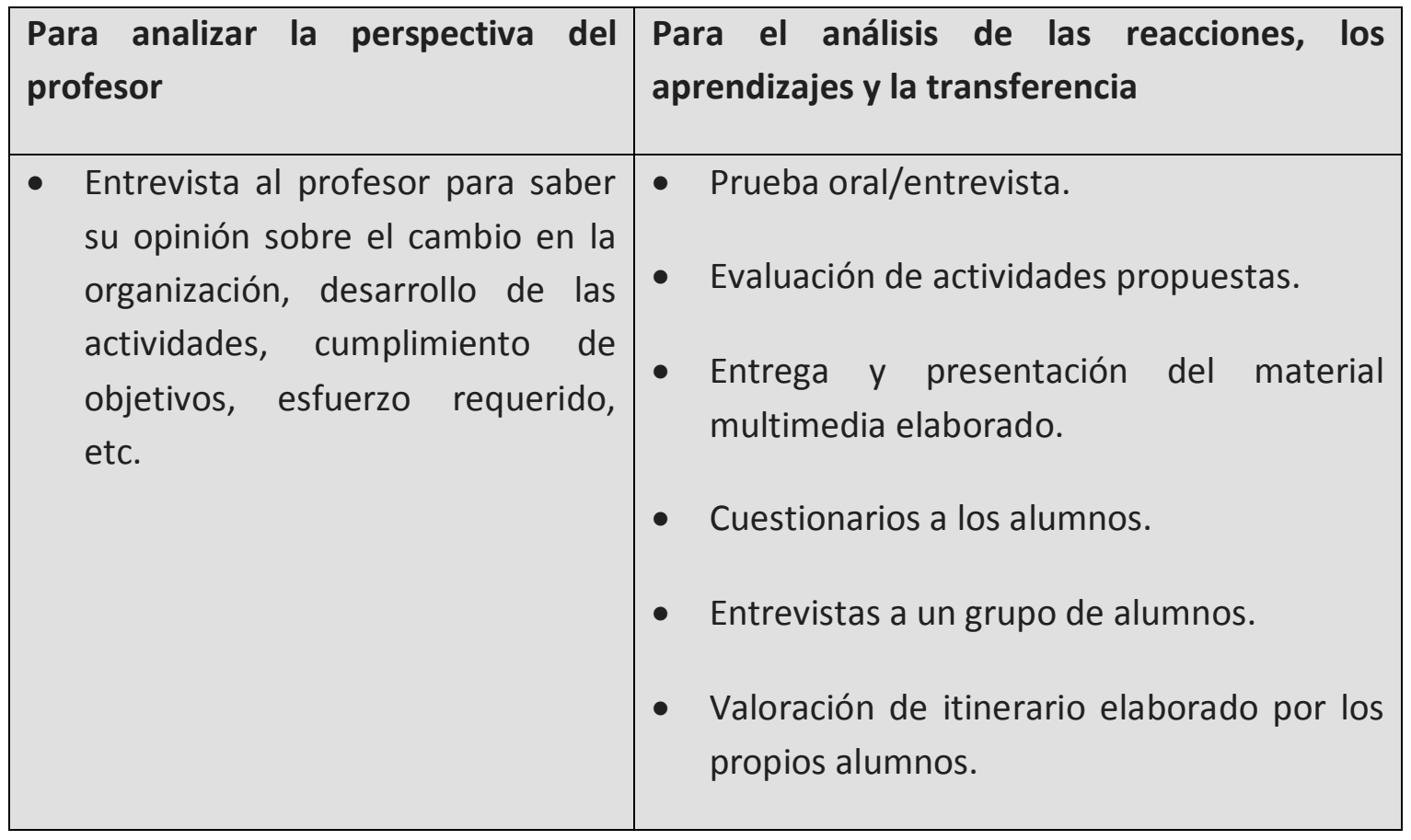

Tabla 2. Instrumentos aplicados en el estudio

La población estaba constituída por los 110 alumnos matriculados en la asignatura, 15 de ellos fueron seleccionados de forma aleatoria para hacerles una entrevista personal, 66 participaron en los cuestionarios y se hizo una entrevista a las dos profesoras.

- Quinta. Análisis de los resultados.

- Sexta. Producción de documentación. Elaboración del informe final y difusión de la investigación.

\section{RESULTADOS}

Los resultados obtenidos se han basado principalmente en la valoración realizada por los alumnos en torno a los siguientes ejes:

- La utilización del itinerario de aprendizaje experto (incluye la metodología para trabajar los contenidos incluidos en el itinerario, aspectos relacionados con el 
aprendizaje y el control que el alumnado tienen sobre su aprendizaje, la utilización de los mapas conceptuales como organizadores de los recursos de aprendizaje incluidos)

- La utilización de los itinerarios de aprendizaje, elaborados por los propios alumnos, como instrumento de apoyo al aprendizaje y para validar y evaluar el proceso de aprendizaje.

A partir de las dos fases de estudio, se obtuvieron resultados y propuestas tanto orientados a los aspectos metodológicos de la materia, como los referidos concretamente al itinerario de aprendizaje diseñado.

\section{Resultados referidos a los aspectos generales de la metodología didáctica}

Uno de los elementos derivados de la primera fase hace referencia a la importancia de atender a los aspectos relacionados con la asignatura en general y no solamente al itinerario de aprendizaje y la implantación de éste en un grupo de alumnos. Surge la necesidad de trabajarlos como tema transversal durante el transcurso de toda la asignatura en lugar de trabajar el módulo de forma aislada, por lo que conlleva cambios en la metodología (de Benito, Darder, Salinas \& Cañas, 2010; Darder, de Benito, Escandell \& Salinas, 2010 y Darder, de Benito, Beltrán \& Bosch, 2010.)

También se observó que si se pretendía que el alumno aprendiese significativamente debía haber cambios, además de en la metodología del docente, en la actitud del alumno. Según Novak esta no es una tarea fácil, ya que los alumnos universitarios llevan muchos años acostumbrados a una enseñanza tradicional y memorística y no es fácil persuadirlos de que acepten las estrategias del aprendizaje significativo, y revisen su manera de aprender.

En general la valoración que hacen los alumnos de la experiencia a través del cuestionario es que es positiva, alrededor del $40 \%$ en ambas fases la valoran como positiva o muy positiva y entre el $28 \%$ y $32 \%$ se muestran indecisos.

Se ha constatado que es un tipo de metodología que según la percepción del alumnado requiere más esfuerzo y no están acostumbrados a la presentación de la información en este formato y al grado de autonomía que requiere (de Benito, B., Salinas, J., Darder, A. \& Marín, V (2011).

La diferencia entre la primera y segunda fase en cuanto a valoración general, las diferencias no son muy notables: sí se ve una disminución de porcentajes con una opinión negativa. Un ejemplo de ello sería la valoración de la experiencia de trabajo y el estudio como positivo, ya que ha disminuido el porcentaje de alumnado que lo valoraba de forma negativa (de un $31.25 \%$ a un $14 \%$ ), ha aumentado los que lo consideran de forma positiva (de un $40.62 \%$ a un $44 \%$ )y de igual forma, ha aumentado el porcentaje de alumnado que lo valora de forma media (de un $28.12 \%$ a un $42 \%$ ). 
Si nos atenemos a las entrevistas, en la primera fase de implementación las docentes percibían la experiencia como positiva, pero debía mejorar la metodología teniendo en cuenta el grupo de alumnos (perfil, motivación, etc.) a través de la concreción de las pautas de trabajo, temporalizando y marcando la realización de actividades intermedias, ya que, explican las docentes, los alumnos no están acostumbrados al nivel de autonomía que implicada el estudio del tema a través de esta metodología.

En relación a esto, los alumnos participantes en el estudio de la segunda fase manifestaron no haber tenido problemas y valorarla positivamente. En las entrevistas realizadas a las profesoras durante esta misma fase, se percibe que la experiencia también ha sido buena, ya que el material multimedia realizado por el alumnado es mejor que el del año anterior.

La metodología utilizada ha sido valorada más positivamente en la segunda fase, un ejemplo de ello son los resultados obtenidos ante la afirmación "Me ha gustado la manera como se han organizado y presentado los contenidos del módulo" los resultados han sido más positivos durante la segunda fase que durante la primera, así, de un $43.75 \%$ que lo valoraba de forma negativa se ha pasado a un $30 \%$, de un $28.12 \%$ que lo veía de forma positiva en la primera fase, encontramos un $35 \%$ en la segunda; $y$, de un $28.12 \%$ que le era indiferente o lo valoraba de forma media, encontramos un $35 \%$ en la segunda fase.

En cuanto a las entrevistas, se observa también mejoría en la opinión de los alumnos, ya que en esta segunda fase, y ya establecidas las pautas de trabajo y el seguimiento a los alumnos por parte de las profesoras, las opiniones son diferentes a las de los alumnos de la primera fase. Así, de las entrevistas al alumnado, se deduce que éstos han considerado la organización del Módulo III y concretamente sus contenidos adecuada y que los recursos disponibles son acertados, porque les han permitido desarrollar el material multimedia progresivamente, a pesar de ser un módulo que implica mucho trabajo y mucha dedicación.

\section{Resultados relacionados con el aprendizaje y el control que el alumnado tienen sobre su aprendizaje}

Con relación al control sobre el aprendizaje, se pretendía averiguar si la metodología y la organización de los contenidos mediante mapas conceptuales provocaban en el alumnado que tuviese un mayor control sobre su proceso de aprendizaje. Se observa, en este caso, que de la primera a la segunda fase hay una diferencia significativa en cuanto a la opinión sobre este tema. En la primera fase, el alumnado creía mayoritariamente que sí que tenía más control sobre su aprendizaje (71.87\% respondió 4 o 5), mientras que en la segunda fase ha disminuido considerablemente (45\%). Mientras que en la primera fase un $6.25 \%$ opinaba que no tenía control sobre su aprendizaje, en esta segunda es un $23 \%$, por tanto, la cifra ha aumentado. Finalmente, los indecisos también han aumentado, de un $21.87 \%$ a un 32\%.

De las entrevistas al alumnado puede resaltarse la diferente opinión que tienen los alumnos sobre el aprendizaje a través de esta metodología. Así, los alumnos entrevistados en la primera fase, en su gran mayoría manifestaron haber aprendido pero no por la metodología 
que se ha seguido sino porque han buscado información del año anterior o por toda la documentación que estaba enlazada en el mapa conceptual. Por el contrario, el alumnado participante en la segunda fase consideraron que habían aprendido y tenido más libertad y más control sobre su aprendizaje, aunque algunos no sentían tener ese control a pesar de haber aprendido más que en otras asignaturas.

\section{Resultados sobre la utilización de los mapas conceptuales como organizadores de los recursos de aprendizaje.}

En cuanto a las opiniones sobre la utilización de los mapas conceptuales como organizadores de los materiales y secuenciación del aprendizaje, en ambas fases consideran que el material es un buen recurso para el autoaprendizaje (un $62.5 \%$ en la primera fase y un $61 \%$ en la segunda han marcado o un 4 o un 5), consideran que el sistema de navegación por los contenidos ha facilitado la orientación, ya que en la primera un $43.75 \%$ opinaba que sí y en la segunda fase encontramos un $61 \%$. Por el contrario, ante la afirmación "El material me ha resultado motivador en su uso y aprendizaje" los resultados no son tan positivos, ya que el porcentaje que opina que sí que es un material motivador (un $37.5 \%$ en la primera fase, frente al $32 \%$ de la segunda) disminuye y aumenta considerablemente el número de indecisos (del $12.5 \%$ de la primera fase, encontramos un $47 \%$ en la segunda fase). Es destacable también que disminuye el porcentaje de encuestados que no están de acuerdo con la afirmación, así en la primera fase era un 50\% del alumnado, y en la segunda solamente es un $21 \%$.

Resultados sobre la utilización de los itinerarios de aprendizaje, elaborados por los propios alumnos, como instrumento de apoyo al aprendizaje y para validar y evaluar el proceso de aprendizaje.

Según las respuestas obtenidas por los alumnos participantes en la segunda fase del estudio, el $49 \%$ de los alumnos/as está de acuerdo o muy de acuerdo en que realizar el itinerario de aprendizaje les ha ayudado a estructurar los contenidos de la asignatura, el $27 \%$ se muetra indiferente, frente al $24 \%$ que están en desacuardo o muy en desacuerdo.

Por lo que respecta a las opiniones del alumnado sobre si el itinerario de aprendizaje favorece el aprendizaje significativo, los resultados son bastante positivos, ya que un $55 \%$ ha respondido que sí frente al $15 \%$ que no lo considera así.

Otro aspecto a tener en cuenta, es que realizar el itinerario de aprendizaje y que éste sea utilizado como herramienta de evaluación, les ha proporcionado el control en el proceso de aprendizaje al $48 \%$ de los encuestados han manifestado estar de acuerdo o muy de acuerdo mientras que el $29 \%$ se han mostrado indiferentes.

Según las entrevistas realizadas a los alumnos, el sistema de evaluación continua es uno de los aspectos que más ha gustado ya que lo relacionan con no hacer examen final, no les importa trabajar cada día a cambio de no tener que hacer examen. Sin embargo, no están de acuerdo en que en un sistema de evaluación continúa la entrevista personal (a la cual 
denominan examen oral) sea tan relevante incluso más que el resto de herramientas de evaluación.

Todos los alumnos entrevistados han elegido hacer evaluación continua y según han manifestado es más motivadora y acorde a la metodología de trabajo utilizada por esta asignatura siempre y cuando implique no hacer examen final.

En el análisis realizado de las entrevistas de las profesoras se observa que hacer una evaluación continua supone mucho trabajo. Ambas profesoras coinciden en que los alumnos no están acostumbrados a trabajar de forma constante sino hacerlo todo para el final, porque aún utilizan la metodología memorística y no hacen un aprendizaje significativo, este es el motivo de que no hayan sabido realizar la entrevista oral (una de las herramientas de evaluación llevada a cabo por las profesoras a los alumnos) pero aún así lo valoran positivamente.

\section{DISCUSIÓN}

El análisis de la aplicación de los itinerarios de aprendizaje durante las dos fases del estudio han proporcionado elementos de reflexión sobre las posibilidades de estas herramientas como elementos para la innovación didáctica.

Por lo que se refiere a su utilización como recurso para la representación del conocimiento, los itinerarios de aprendizaje representados en mapas conceptuales presentan una doble capacidad de representación. Por un lado, permite jerarquizar niveles sucesivos de complejidad (representación en "espiral" en niveles de elaboración), al mismo tiempo que se presenta como mapa de experto, dado que facilita la integración en un mismo soporte de diferentes técnicas para representar contenidos.

En una primera instancia el control sobre la organización de la asignatura, los contenidos y los objetos de aprendizaje recae sobre el profesor, ya que se trata de proporcionar un itinerario de aprendizaje experto y por lo tanto le ofrece la flexibilidad suficiente como para organizar estos elementos como él considere conveniente. Pero la utilización de los mapas conceptuales como herramienta para construir el itinerario no sólo ofrece flexibilidad al profesor sino también a los alumnos ya que les da control sobre su proceso de aprendizaje y al poder modificarlo el alumno puede construir su propio itinerario de aprendizaje. En este sentido los itinerarios de aprendizaje, nos facilita la utilización de metodologías centradas en el alumno ya que plantean una secuencia no lineal, lo que permite estructurar y secuenciar los contenidos, obteniendo modelos que van desde el control total del docente sobre la secuencia que sigue el alumno hasta el total control del alumno sobre dicha secuencia.

Los mapas conceptuales se evidencian como una potente herramienta para la estructuración y presentación de la información y recursos de aprendizaje. Proporcionan flexibilidad frente a otras herramientas que suelen ser más rígidas a la hora de organizar contenidos obligando a los docentes a organizarlos de manera lineal, mostrando como único organizador previo un 
índice que no muestra ningún tipo de relaciones entre los contenidos. En este sentido y de acuerdo con Ausubel, Novak y Hanesian (1983), los itinerarios de aprendizaje mediante mapas conceptuales actúan como verdaderos organizadores previos al proporcionar "andamiaje ideacional", servir de apoyo al alumno frente a la nueva información actuando de puente entre el conocimiento actual del alumno y el nuevo material.

De esta forma, itinerario constituye algo más que un organizador de contenidos, viniendo a ser un organizador de entornos de enseñanza-aprendizaje desde el momento en que facilitan una secuencia no lineal del aprendizaje y la organización de Objetos de Aprendizaje. Y en esta línea de trabajo es en la que consideramos debería profundizar nuestra investigación.

La metodología utilizada durante toda la asignatura (la construcción de su propio itinerario, la realización de actividades periódicas, la planificación de esta, la elección del sistema de evaluación, etc.) permite participar activamente al alumnado en su aprendizaje, y de esta forma aumentan las posibilidades de que el aprendizaje sea significativo.

Sin embargo, la implementación de itinerarios de aprendizaje requiere, desde el punto de vista metodológico, una detallada planificación, así como actividades de seguimiento continuo por parte del docente. Esta fue una de las conclusiones surgidas de la primera fase, y corroborada en la segunda, ya que los resultados han constatado que las actividades y planificación de éstas referidas a la materia del itinerario han ayudado al aprendizaje, aunque, por otra parte, la propuesta de actividades tan pautada pueda haber provocado en los alumnos la sensación de pérdida de control sobre el aprendizaje.

Este tipo de metodología se adapta a los modelos de enseñanza flexible, facilitando la organización y secuenciación de los contenidos, proporcionando orientación y guía sobre éstos. Ello requiere profesorado cuyo desempoeño profesional esté acorde a los postulados de dicho modelo pero también alumnos preparados para seguirlo.

En relación con la preparación del alumnado, otra conclusión de la primera fase fue que utilizar itinerarios de aprendizaje basados en mapas conceptuales requiere que éste posea destrezas para la creación de mapas conceptuales, y así queda reflejado en los resultados de la segunda fase, en los que se observa que una gran mayoría cree que la realización de un taller de mapas conceptuales ha sido una buena base para poder trabajar con el itinerario experto y con su propio itinerario.

La experiencia realizada nos ha abierto nuevas líneas de investigación relacionadas con el tema entre las que destacamos:

- La utilización de los mapas conceptuales para la representación del conocimiento de los alumnos y por lo tanto como herramienta de evaluación.

- Los mapas conceptuales como organizadores del conocimiento y soporte para la creación de itinerarios personales de aprendizaje. Este tema está estrechamente relacionado con el porfolio. 
- Utilización de los mapas conceptuales como herramienta de creación compartida de conocimiento apoyando el trabajo colaborativo.

- Los mapas conceptuales como organizadores gráficos de los materiales u objetos de aprendizaje.

\section{REFERENCIAS BIBLIOGRAFICAS}

AUSUBEL, D. P.; NOVAK, J. D. y HANESIAN, H. (1983). Psicología educativa: un punto de vista cognitivo. México, Editorial Trillas.

DARDER, A, DE BENITO, B., BERTRÁN G, BOSCH, M. (2010): Los itinerarios de aprendizaje mediante mapas conceptuales como recurso para la representación del conocimiento. Comunicación en el Congreso Internacional EDUTEC 2010: E-Learning 2.0. Enseñar y Aprender en la Sociedad del Conocimiento. Bilbao. Accesible desde: http://gte.uib.es/pape/gte/sites/gte.uib.es.pape.gte/files/LOS\%20ITINERARIOS\%20DE\%2 OAPRENDIZAJE\%20MEDIANTE\%20MAPAS\%20CONCEPTUALES\%20COMO\%20RECURSO\%2 OPARA\%20LA\%2OREPRESENTACION.pdf

DARDER, A, DE BENITO, B., ESCANDELL, C. SALINAS, J. (2010): Construcción, validación e implementación de un itinerario de aprendizaje basado en mapas conceptuales. Comunicación en el Congreso Internacional EDUTEC 2010: E-Learning 2.0. Enseñar y Aprender en la Sociedad del Conocimiento. Bilbao. Accesible desde: http://gte.uib.es/pape/gte/sites/gte.uib.es.pape.gte/files/Construccion, $\% 20$ validacion $\% 2$ 0e\%20implementacion\%20de\%20un\%20itinerario\%20de\%20aprendizaje\%20mediante\%2 Omapas.pdf

DE BENITO, B. (2000). Herramientas para la creación, distribución y gestión de cursos a través de Internet. Edutec. Revista Electrónica de Tecnología Educativa. Num. 12. Recuperado el 16/11/11 de http://edutec.rediris.es/Revelec2/Revelec12/PDF/deBenito.pdf

DE BENITO, B., DARDER A, SALINAS, J, CAÑAS, A (2010): Construcción y validación de un itinerario de aprendizaje sobre diseño y producción de materiales didácticos multimedia. Póster presentado en Congreso CMC 2010 - 40 Congreso Internacional Sobre Mapas Conceptuales, Viña del Mar, Chile - Octubre 5, 6, 7, 2010. Recuperado el 2/12/11 de http://cmc.ihmc.us/cmc2010papers/cmc2010-84.pdf

DE BENITO, B., SALINAS, J., DARDER, A. \& MARÍN, V (2011): Los itinerarios de aprendizaje a través de mapas conceptuales como estrategia de innovación metodológica. Comunicación en el Congreso Internacional EDUTEC 2011: Formación Docente en Entornos Virtuales para la Transformación del Aprendizaje. Pachuca, Hidalgo (México) Accesible desde: http://gte.uib.es/pape/gte/sites/gte.uib.es.pape.gte/files/itinerarios aprendizaje.pdf 
GONZÁLEZ, F.M (2008). El Mapa Conceptual y el Diagrama V. Recursos para la Enseñanza Superior en el siglo XXI. Madrid: Narcea S.A, de Ediciones. ISBN: 978-84-277-1573-8

KIRKPATRICK, D. (1994): Evaluation Training Programs: The Four Levels. Berrett-Kohler San Francisco

NOVAK, J.D., (1998): Learning, Creating and Using Knowledge. Concept Maps as Faccilitative Tools in Schools and Corporations. Lawrence Erlbaum As. Mahwah NJ.

REEVES, T. C. (2000). Enhancing the Worth of Instructional Technology Research through "Design Experiments" and Other Development Research Strategies. International Perspectives on Instructional Technology Research for the 21st Century Symposium. New Orleans, LA, USA.

REIGELUTH, C.M (1995). What is the New Paradigm of Instructional Theory?. ITForum Paper. Recuperado el 16/06/2011 de http://itechl.coe.uga.edu/itforum/paper17/paper17.html

SALINAS, J (2004). Innovación docente y uso de las TIC en la enseñanza universitaria. Revista de Universidad y Sociedad del Conocimiento (RUSC). UOC, Vol 1, no1. Recuperado el 17/06/11 de http://www.uoc.edu/rusc/dt/esp/salinas1104.pdf

SALINAS, J.; DE BENITO, B. Y DARDER, A. (2011). Los mapas conceptuales como organizadores del proceso de enseñanza-aprendizaje: los itinerarios de aprendizaje. IN. Revista Electrònica d'Investigació i Innovació Educativa i Socioeducativa, V. 3, n. 1, pag. 63-74. Recuperado el 16/11/11 de http://www.uib.es/ant/infsobre/estructura/instituts/ICE/revista_IN/pags/volumenes/vol 3 num1/revista/salinasyotros.pdf

\section{Para citar este artículo:}

DE BENITO, B. DARDER, A. \& SALINAS, J. (2012). Los itinerarios de aprendizaje mediante mapas conceptuales como recurso para la representación del conocimiento. Edutec, Revista Electrónica de Tecnología Educativa, 39. Recuperado el dd/mm/aa de http://edutec.rediris.es/Revelec2/Revelec39/itinerarios aprendizaje mapas conceptuales $r$ epresentacion conocimiento.html 